\title{
Dynamical polaron Ansatz: A theoretical tool for the ultrastrong-coupling regime of circuit QED
}

\author{
Guillermo Díaz-Camacho, Alejandro Bermudez, and Juan José García-Ripoll \\ Instituto de Física Fundamental, Consejo Superior de Investigaciones Científicas, Calle Serrano 113b, Madrid 28006, Spain
}

(Received 7 January 2016; published 25 April 2016)

\begin{abstract}
In this work we develop a semianalytical variational Ansatz to study the properties of few-photon excitations interacting with a collection of quantum emitters in regimes that go beyond the rotating-wave approximation. This method can be used to approximate both the static and dynamical properties of a superconducting qubit in an open transmission line, including the spontaneous emission spectrum and the resonances in scattering experiments. The approximations are quantitatively accurate for rather strong couplings, as shown by a direct comparison to matrix-product-state numerical methods, and provide also a good qualitative description for stronger couplings well beyond the Markovian regime.
\end{abstract}

DOI: 10.1103/PhysRevA.93.043843

\section{INTRODUCTION}

The impressive progress in coupling single photons to single emitters, both in the microwave [1] and in the optical domains [2], allows us to talk about an emerging field of propagating-photon quantum technology. This field has already demonstrated new devices that predict interesting applications, such as few-photon transistors [3,4], nonclassical states of the radiation field $[5,6]$, or photon-mediated interactions $[7,8]$.

The development of these technologies has been accompanied by numerous analytical and numerical techniques to model light-matter and light-mediated-matter interactions. These include single-photon single-qubit effective boundary conditions $[9,10]$, input-output theory $[11,12]$, and scattering theory [13-15]. Nonetheless, the degree of control of some methods, or their computational generality for arbitrary numbers of photons, remains an open problem. Moreover, all these methods are restricted to the rotating-wave approximation (RWA) regime, in which counterrotating terms are neglected. The RWA breaks down, for instance, when the spontaneous emission rate of a two-level quantum emitter $\gamma$ becomes comparable to its energy gap $\Delta \sim \gamma$. Such deep ultrastrong coupling is now within experimental reach in the superconducting world [16-19] and we expect that it will also become feasible in the near future in experiments with single emitters in contact with optical photons and plasmons.

The microscopic Hamiltonian describing light-matter interactions can be formally mapped, in any coupling regime, onto the so-called spin-boson model [20]. Hence, in dealing with strong and ultrastrong interactions, optics can look into condensed-matter physics for inspiration, generalizing methods that work with the spin-boson Hamiltonians and adapting them in order to describe few propagating photon excitations. Some of these methods, such as the noninteracting-blip approximation [20] or Wilson's numerical renormalization group [21], focus directly on the dynamics of the emitters, such that one cannot address the scattering of photons. Other methods, such as the sophisticated numerical techniques based on the density-matrix renormalization group (DMRG) or matrix-product states (MPSs), have a greater potential in dealing with these effects $[19,22,23]$, at the expense of a higher computational cost.

In this work we study a third family of methods based on the Lang-Firsov transformation for the polaron problem
[24]. These methods consist of a variationally optimized unitary transformation that displaces the electromagnetic field based on the state of the two-level emitter and have already provided valuable results for the equilibrium properties of the spin-boson model [25-27]. We introduce a dynamical polaron Ansatz, which is a time-dependent variational wave function describing the two-level emitter at zero temperature, together with the scattering states of the photons. We thus upgrade this family of variational methods to address relevant nonequilibrium problems, such as the spontaneous emission or the ultrastrong spectroscopy of the emitter. Additionally, these dynamical Ansätze lead to analytical results in certain regimes, which are valuable to develop a physical intuition about these complex nonequilibrium effects and can be straightforwardly generalized to more complex situations with multiple quantum emitters coupled to propagating photons. The numerical and analytical methods are simpler than full MPS simulations and a comparison with these shows good qualitative and even quantitative agreement up to very large coupling strengths $\gamma \sim 0.4 \Delta$ in the Ohmic spin-boson model. This shows the potential of these conceptually and technically simpler tools for analyzing and designing future experiments in propagatingphoton quantum technologies.

The outline of this work is as follows. In Sec. II we introduce the spin-boson model as we use it to describe lightmatter interaction of a few two-level emitters, also referred to as qubits, with a low-dimensional photonic waveguide or microwave transmission line. In Sec. III we describe different variational Ansätze. We start with two variational Ansätze based on the Lang-Firsov transformation describing a static and a time-dependent wave function with up to one hybrid excitation with variational weights in the qubits or in the photonic modes. We also describe briefly the MPS methods that we compare with ours. In Sec. IV we apply all these methods to the study of a single quantum emitter in the photonic line and its interaction with the propagating photons. We show how the Ansatz properly describes the qubit polarization in the open field, the frequency renormalization, and also the transmission and reflection coefficients for an incoming low-intensity photonic wave packet with a broad distribution among the possible photonic modes, even in the ultrastrong-coupling regime. Finally, in Sec. V we summarize our work and discuss our conclusions and possible extensions to treat multiple quantum emitters. 


\section{SPIN-BOSON MODEL}

The Hamiltonian describing a collection of quantum emitters interacting with a one-dimensional electromagnetic (em) field in the ultrastrong-coupling regime corresponds to the well-known few-impurity spin-boson model [20,28], unitarily transformed to a rotated spin basis, namely,

$$
H=\sum_{i} \frac{\Delta_{i}}{2} \sigma_{i}^{z}+\sum_{k} \omega_{k} a_{k}^{\dagger} a_{k}+\sum_{i k} \sigma_{i}^{x}\left(g_{i k} a_{k}^{\dagger}+\text { H.c. }\right) .
$$

Here $a_{k}^{\dagger}$ and $a_{k}$ are bosonic operators that create and annihilate a quantum of the em field with frequency $\omega_{k}$ labeled by $k$ (e.g., in three dimensions, $k$ contains the photon wave vector and its polarization). The emitters are modeled as two-level atoms, also referred to as qubits or spins, $\sigma_{i}^{z}=\left|\uparrow_{i}\right\rangle\left\langle\uparrow_{i}|-| \downarrow_{i}\right\rangle\left\langle\downarrow_{i}\right|$, $\sigma_{i}^{+}=\left|\uparrow_{i}\right\rangle\left\langle\downarrow_{i}\left|, \sigma_{i}^{-}=\right| \downarrow_{i}\right\rangle\left\langle\uparrow_{i}\right|$, and $\sigma_{i}^{x}=\sigma_{i}^{+}+\sigma_{i}^{-}$, and we have introduced the transition frequency $\Delta_{i}$.

In the above Hamiltonian, the atom-photon interaction is defined in terms of the couplings $g_{i k}$, which depend on the positions of the qubits $\boldsymbol{x}_{i}$. As customary in the quantum theory of radiation [29], the em field acts as a bosonic reservoir that modifies the dynamics of the quantum emitters depending on the distribution of the atom-photon couplings for different frequencies, namely, the spectral density

$$
J_{i}(\omega)=2 \pi \sum_{k}\left|g_{i k}\right|^{2} \delta\left(\omega-\omega_{k}\right)
$$

For instance, when the atom-photon couplings are weak and the correlations of the em bath decay sufficiently fast, a single excited qubit in contact with the em field will decay exponentially with a rate $\gamma_{i}=J_{i}(\Delta)$ [30], while a pair of qubits will show collective effects due to the exchange [31] or the spontaneous emission [32] of em photons.

We consider both discrete and continuous descriptions of a one-dimensional em reservoir, which model the photons in a transmission line.

(i) Discretized spin-boson model. A transmission line of length $L$ can be divided into $N$ segments of length $\delta x=L / N$, which leads to a discretized momentum $k_{n}=\frac{2 \pi}{L} n$, with $n \in\{0, \pm 1, \ldots, \pm N / 2\}$. The boson frequencies $\omega_{k} \rightarrow \omega_{k_{n}}$, which arise due to the couplings between the transmission line segments, and the spin-boson couplings $g_{i k} \rightarrow g_{i k_{n}}$ correspond to

$$
\omega_{k_{n}}=\omega_{\mathrm{c}} \sqrt{2-2 \cos \left(\frac{2 \pi n}{N}\right)}, \quad g_{i k_{n}}=g \sqrt{\frac{\omega_{k_{n}}}{2 L}} e^{i(2 \pi / n L) x_{i}} .
$$

Here we have introduced the cutoff frequency $\omega_{\mathrm{c}}=v / \delta x$, which increases as the number of segments is raised. In the continuum limit $N \rightarrow \infty$ and for energies well below the cutoff, one recovers the linear dispersion $\omega_{k} \sim v|k|$, with $v$ playing the role of the speed of light in the transmission line. Let us note that this discretized model will be used to perform numerical calculations.

(ii) Continuum spin-boson model. Alternatively, we can model the transmission line directly in the continuum limit $\delta x \rightarrow 0$, where $k \in \mathbb{R}$, and the high-frequencies are exponen- tially cut off by substituting

$$
\omega_{k}=v|k|, \quad g_{i k}=g e^{-\omega_{k} / 2 \omega_{\mathrm{c}}} \sqrt{\frac{\omega_{k}}{2 L}} e^{i k x_{i}} .
$$

Let us note that the cutoff in this model can be set to any desired value and the dispersion is assumed to be linear for all frequencies. This continuous model will be exploited to derive a number of analytical predictions.

Both models of the bosonic bath lead to an Ohmic spectral density at low-enough frequencies $\omega \ll \omega_{\mathrm{c}}$, which is identical for all qubits, namely,

$$
J(\omega):=J_{i}(\omega) \approx \pi \alpha \omega, \quad \alpha=\frac{|g|^{2}}{\pi v},
$$

where $\alpha$ is a dimensionless spin-boson coupling strength that plays an important role. In the context of the single-impurity spin-boson model, ultrastrong couplings lead to the so-called localization-delocalization quantum phase transition $\alpha=1$ $[33,34]$ and the coherent-incoherent dynamical crossover at $\alpha=1 / 2[35,36]$.

As mentioned above, we explore the consequences of such effects in the static and dynamical properties for a few quantum emitters (1) by using two types of variational Ansätze.

(i) Polaron variational Ansatz. This ansatz was originally developed to understand the ground-state properties of the Kondo effect through its connection to the spin-boson model $[25,26]$ and has the nonvariational Lang-Firsov transformation as its precursor [24]. The polaron ansatz builds on a variational family of spin-dependent coherent states and correctly captures the quantum phase transition at $\alpha=1$. As shown in [27], the polaron method also agrees with some of the finitetemperature properties obtained by other methods [20] and can be combined with Markovian master equations to study the spin dynamics, although the predicted coherent-incoherent crossover does not coincide with $\alpha=1 / 2$. In this work we introduce a dynamical polaron Ansatz that will allow us to overcome these limitations and to develop a simple analytical understanding of how the archetypical quantumoptical properties of (1) predicted within the RWA [30-32] are modified in the ultrastrong-coupling regime. Moreover, this Ansatz will allow us to address the dynamics of the propagating photons, which is extremely important given their experimental accessibility.

(ii) Matrix-product-state variational Ansatz. Introduced in Ref. [37] and connected to the DMRG [38] for onedimensional quantum systems in [39], MPS Ansätze have the nonvariational valence-bond states as their precursor [40]. The MPS Ansatz is defined as the product of a set of matrices, whose number is determined by the structure of the problem, and its size determines the accuracy of the variational procedure. As explained below, in this work we use static and time-dependent MPSs to study the spin-boson model in frequency space, generalizing static and time-dependent techniques introduced in [19].

The comparison between the quasiexact numerical Ansatz of MPSs and the dynamical polaron Ansatz applied to Eq. (1) will allow us to assert the regimes of validity of the latter. In our effort to make the polaron Ansatz more familiar to the quantum optics community interested in going beyond 
the weak-coupling RWA regime, we determine the regimes of validity of its simpler analytical predictions.

\section{VARIATIONAL METHODS FOR THE ULTRASTRONG-COUPLING REGIME}

In this section we describe in detail the above variational techniques that can be used to study both static and dynamical effects for a collection of quantum emitters interacting with a one-dimensional (1D) em field through the spin-boson model (1). We describe the general approach and leave its application to particular settings for the following sections.

\section{A. Static polaron Ansatz}

The static polaron Ansatz is a variational method to approximate the equilibrium properties of a single-impurity spin-boson model, which has been applied to the Ohmic [25-27] and sub-Ohmic [41] cases with significant success, in particular in light of its considerable simplicity. It can be improved by enlarging the number of variational parameters [42] and generalized to the two-impurity spin-boson model [43].

The static Ansatz can be defined in terms of a variational polaron transformation, which captures the relevant correlations between the spins and the bosons and acts on a product state where the spins and the bosons are not entangled. For an arbitrary number of spins $N_{\mathrm{s}}$, it can be defined as

$$
\left|\Psi_{\text {g.s. }}^{\mathrm{P}}\left[f_{i k}, c_{\sigma}\right]\right\rangle=U_{\mathrm{P}}^{\dagger}\left[f_{i k}\right]|0\rangle \otimes\left|\psi_{\mathrm{s}}\left[c_{\sigma}\right]\right\rangle .
$$

Here we have introduced the polaron unitary transformation

$$
U_{\mathrm{P}}\left[f_{i k}\right]=\bigotimes_{i, k} e^{\sigma_{i}^{x}\left(f_{i k}^{*} a_{k}^{\dagger}-\text { H.c. }\right)},
$$

where $\left[f_{i k}\right]$ is the set of all variational polaron parameters $f_{i k} \in \mathbb{C}$. The static polaron ansatz (6) is defined in terms of the global bosonic vacuum $|0\rangle$ and the variational spin state

$$
\left|\psi_{\mathrm{s}}\left[c_{\sigma}\right]\right\rangle=\sum_{\sigma \in\{\uparrow, \downarrow\}^{N_{\mathrm{s}}}} c_{\sigma}\left|\sigma_{1}\right\rangle \otimes\left|\sigma_{2}\right\rangle \otimes \cdots \otimes\left|\sigma_{N_{\mathrm{s}}}\right\rangle,
$$

which depends on the set $\left[c_{\sigma}\right]$ of all variational spin parameters $c_{\sigma}=c_{\sigma_{1}, \sigma_{2}, \ldots, \sigma_{N_{\mathrm{S}}}} \in \mathbb{C}$, fulfilling $\sum_{\sigma}\left|c_{\sigma}\right|^{2}=1$.

The variational minimization over the spin-boson Hamiltonian (1), defined as

$$
\epsilon_{\text {g.s. }}^{\mathrm{P}}=\min _{\left[f_{i k}, c_{\sigma}\right]}\left\{\left\langle\Psi_{\text {g.s. }}^{\mathrm{P}}\left[f_{i k}, c_{\sigma}\right]|H| \Psi_{\text {g.s. }}^{\mathrm{P}}\left[f_{i k}, c_{\sigma}\right]\right\rangle\right\},
$$

can be expressed in terms of a simpler minimization

$$
\epsilon_{\text {g.s. }}^{\mathrm{P}}=\min _{\left[f_{i k}, c_{\sigma}\right]}\left\{\left\langle\psi_{\mathrm{s}}\left[c_{\sigma}\right]\left|H_{\mathrm{s}}\left[f_{i k}\right]\right| \psi_{\mathrm{s}}\left[c_{\sigma}\right]\right\rangle\right\},
$$

which requires diagonalizing the following spin Hamiltonian instead of the original spin-boson model:

$$
H_{\mathrm{s}}\left[f_{i k}\right]=\sum_{i} \frac{\Delta_{i}}{2} e^{-\Xi_{i}\left[f_{i k}\right]} \sigma_{i}^{z}+\sum_{i, j} J_{i j}\left[f_{i k}\right] \sigma_{i}^{x} \sigma_{j}^{x} .
$$

This spin Hamiltonian corresponds to a long-range version of the paradigmatic Ising model in a transverse field [44]. This model displays qubit frequencies that get exponentially renormalized through

$$
\Xi_{i}\left[f_{i k}\right]=\sum_{k} f_{i k} f_{i k}^{*}+\text { c.c. }
$$

and photon-mediated Ising interactions with strengths

$$
J_{i j}\left[f_{i k}\right]=\sum_{k}\left(\omega_{k} f_{i k} f_{j k}^{*}-g_{i k} f_{j k}^{*}-g_{i k}^{*} f_{j k}\right) .
$$

Since the variational energy is a quadratic functional of the spin parameters, their minimization is simple and can be carried out analytically for the single- or the two-impurity problem, which fixes the spin parameters in terms of the polaron ones. Accordingly, the problem reduces to function minimization and yields the optimal parameters $\left[f_{i k}^{\star}, c_{\sigma}^{\star}\right]$, which are denoted by a star superindex and will be used in the following sections. For more than two impurities, since the Ising interactions can have any particular pattern, an analytical solution cannot be obtained in general and one must resort to Lanczos methods to extract the ground state of the spin Hamiltonian, which can be efficiently implemented for reasonably high $N_{\mathrm{s}}$.

\section{B. Dynamical polaron ansatz}

Although understanding the static properties of the spinboson model is already a nontrivial problem, in particular for a few impurities, a considerably more challenging task is to develop an accurate description of nonequilibrium effects and a number of techniques have been put forth over the years for that purpose [20]. As already mentioned in the Introduction, most of these techniques can only address qubit observables, which is consistent with situations where the bosonic bath cannot be measured. However, with the advent of the new propagating-photon quantum technologies, this situation has been reversed, as the photonic properties of the setup are now accessible. The goal of this section is to introduce an accurate yet simple variational Ansatz that captures these dynamical effects for both the emitters and the photons.

In order to introduce such a dynamical polaron Ansatz, let us revisit the spin Hamiltonian in Eq. (11), specified for the optimal parameters $\left[f_{i k}^{\star}, c_{\sigma}^{\star}\right]$ obtained with the static Ansatz. The eigenstates of this Hamiltonian contain, in addition to the variational ground state $\left|\psi_{\text {g.s. }}\right\rangle:=\left|\psi_{\mathrm{s}}\left[c_{\sigma}^{\star}\right]\right\rangle$, a number $N_{\mathrm{e}}$ of spin excitations $\left\{\left|\psi_{\mathrm{e}}^{s}\right\rangle\right\}$ with energies $\left\{\epsilon_{\mathrm{e}}^{s}\right\}$ that can be excited if the qubits absorb a photon from the em environment. Inspired by our previous works on different quantum manybody models [45], we can define a dynamical variational ansatz by creating such spin and photonic excitations over the polaron-transformed ground state, namely,

$$
\left|\Psi_{\mathrm{exc}}^{\mathrm{P}}\left[\alpha_{s}(t), \alpha_{k}(t)\right]\right\rangle=U_{\mathrm{P}}^{\dagger}\left[f_{i k}^{\star}\right] W_{\mathrm{sp}}\left[\alpha_{s}(t), \alpha_{k}(t)\right]|0\rangle \otimes\left|\psi_{\text {g.s. }}\right\rangle,
$$

where we have introduced an operator that creates the relevant spin-photon excitation

$$
W_{\mathrm{sp}}\left[\alpha_{s}(t), \alpha_{k}(t)\right]=\sum_{s=1}^{N_{\mathrm{e}}} \alpha_{s}(t)\left|\psi_{\mathrm{e}}^{s}\right\rangle\left\langle\psi_{\text {g.s. }}\right|+\sum_{k} \alpha_{k}(t) a_{k}^{\dagger},
$$

with a certain set $\left[\alpha_{s}(t), \alpha_{k}(t)\right]$ of time-dependent variational parameters $\alpha_{s}(t) \in \mathbb{C}, \alpha_{k}(t) \in \mathbb{C}$. 
By rearranging these parameters in a complex-valued vector $\boldsymbol{\alpha}(t)=\left[\alpha_{1}(t), \ldots, \alpha_{N_{\mathrm{e}}}(t), \alpha_{k_{1}}(t), \alpha_{k_{2}}(t), \ldots\right]^{\mathrm{t}}$ fulfilling $\boldsymbol{\alpha}^{\dagger}(t) \boldsymbol{\alpha}(t)=1$, we can construct a Lagrangian that leads to a time-dependent variational principle [46], namely,

$$
\mathscr{L}_{\mathrm{P}}\left[\boldsymbol{\alpha}^{\dagger}, \boldsymbol{\alpha}\right]=\frac{i}{2}\left(\boldsymbol{\alpha}^{\dagger} \partial_{t} \boldsymbol{\alpha}-\partial_{t} \boldsymbol{\alpha}^{\dagger} \boldsymbol{\alpha}\right)-\mathscr{E}_{\mathrm{P}}\left[\boldsymbol{\alpha}^{\dagger}, \boldsymbol{\alpha}\right],
$$

where we do not write the explicit time dependence to ease notation $\boldsymbol{\alpha}=\boldsymbol{\alpha}(t)$. Here we have introduced the energy functional associated with the spin-boson Hamiltonian (1), namely,

$$
\mathscr{E}_{\mathrm{P}}\left[\boldsymbol{\alpha}^{\dagger}, \boldsymbol{\alpha}\right]=\left\langle\Psi_{\mathrm{exc}}^{\mathrm{P}}[\boldsymbol{\alpha}]\left|\left(H-\epsilon_{\text {g.s. }}^{\mathrm{P}}\right)\right| \Psi_{\mathrm{exc}}^{\mathrm{P}}[\boldsymbol{\alpha}]\right\rangle,
$$

which is a quadratic functional of the variational parameters. Building a variational action from the above Lagrangian, the principle of minimal action [46] leads to a system of Euler-Lagrange equations that describes the dynamics of the system restricted to the region of the Hilbert space spanned by the states parametrized by Eq. (14). The accuracy of this variational method thus depends on our physically motivated choice of the dynamical Ansatz (14) and will be benchmarked by comparing it to well-known properties for qubit observables of the single-impurity spin-boson model [20] and to our results of photon scattering using time-dependent MPS simulations.

For the simple parametrization (14), the Lagrangian leads to a linear system of first-order differential equations $i \partial_{t} \boldsymbol{\alpha}=$ $\mathbb{H}_{\mathrm{P}} \boldsymbol{\alpha}$. Here the matrix $\mathbb{H}_{\mathrm{P}}$ can be obtained by evaluating the matrix elements

$$
\left\langle 0\left|\otimes\left\langle\psi_{\text {g.s. }}\left|W_{\text {sp }}^{\dagger}[\boldsymbol{\alpha}]\left(H_{\mathrm{P}}\left[f_{i k}^{\star}\right]-\epsilon_{\text {g.s. }}^{\mathrm{P}}\right) W_{\text {sp }}[\boldsymbol{\alpha}]\right| 0\right\rangle \otimes\right| \psi_{\text {g.s. }}\right\rangle
$$

for the polaron-transformed spin-boson Hamiltonian

$$
\begin{aligned}
H_{\mathrm{P}}\left[f_{i k}^{\star}\right]= & \sum_{i} \frac{\Delta_{i}}{2}\left(\sigma_{i}^{z} \cos \Theta_{i}\left[f_{i k}^{\star}\right]-\sigma_{i}^{y} \sin \Theta_{i}\left[f_{i k}^{\star}\right]\right) \\
& +\sum_{k} \omega_{k} a_{k}^{\dagger} a_{k}+\sum_{i, j} J_{i j}\left[f_{i k}^{\star}\right] \sigma_{i}^{x} \sigma_{j}^{x} \\
& +\sum_{i k} \sigma_{i}^{x}\left[\left(g_{i k}-\omega_{k} f_{i k}^{\star}\right) a_{k}+\text { H.c. }\right]
\end{aligned}
$$

which depends on the operator

$$
\Theta_{i}\left[f_{i k}^{\star}\right]=-2 i \sum_{k}\left(f_{i k}^{\star} a_{k}-\text { H.c. }\right) .
$$

By diagonalizing the matrix $\mathbb{H}_{\mathrm{P}}$, one finds the excitation energies and eigenstates, which are an admixture of the spins and photons and can be understood as some sort of spin-photon waves. However, if one is interested in the reduced dynamics of either the spins, as is customary in studies of the spinboson model [20], or an incoming photonic wave packet for transmission-reflection experiments, one may try to develop a Weisskopf-Wigner-type theory [30] for the above system of first-order differential equations. These equations can always be rewritten as

$$
\begin{aligned}
& i \partial_{t} \alpha_{s}(t)=\Delta_{s} \alpha_{s}(t)+\sum_{k} \mathfrak{g}_{k s} \alpha_{k}(t), \\
& i \partial_{t} \alpha_{k}(t)=\omega_{k} \alpha_{k}(t)+\sum_{s} \mathfrak{g}_{k s}^{*} \alpha_{s}(t)+\sum_{k^{\prime}} \mathfrak{f}_{k k^{\prime}}^{*} \alpha_{k^{\prime}}(t),
\end{aligned}
$$

where we have introduced the energy of the spin excitations $\Delta_{s}=\epsilon_{\mathrm{e}}^{s}-\epsilon_{\mathrm{g} . s .}^{\mathrm{P}}$ and the couplings $\mathfrak{g}_{k s}$, and $\mathfrak{f}_{k k^{\prime}}$, which can be obtained from the matrix elements of $\mathbb{H}_{\mathrm{P}}$. Note that the couplings fulfill $\mathfrak{f}_{k k^{\prime}}=\mathfrak{f}_{k^{\prime} k}$ and can be thus diagonalized by an orthogonal transformation $\Delta \omega_{k} \delta_{k k^{\prime}}=\sum_{k_{1}, k_{1}^{\prime}} \mathscr{M}_{k_{1} k} \mathfrak{f}_{k_{1} k_{1}^{\prime}} \mathscr{M}_{k_{1}^{\prime} k^{\prime}}$. Accordingly, we can transform the variational parameters $\tilde{\alpha}_{k}(t)=\sum_{k^{\prime}} \mathscr{M}_{k^{\prime} k} \alpha_{k^{\prime}}(t)$ and the remaining couplings $\tilde{\mathfrak{g}}_{k s}=$ $\sum_{k^{\prime}} \mathscr{M}_{k^{\prime} k} \mathfrak{g}_{k^{\prime} s}$ such that

$$
\begin{aligned}
& i \partial_{t} \alpha_{s}(t)=\Delta_{\mathrm{r}} \alpha_{s}(t)+\sum_{k} \tilde{\mathfrak{g}}_{k s} \tilde{\alpha}_{k}(t) \\
& i \partial_{t} \tilde{\alpha}_{k}(t)=\left(\omega_{k}+\Delta \omega_{k}\right) \tilde{\alpha}_{k}(t)+\sum_{s} \tilde{\mathfrak{g}}_{k s}^{*} \alpha_{s}(t) .
\end{aligned}
$$

This system of equations resembles the Weisskopf-Wigner equations of spontaneous emission of two-level atoms coupled to the em field [30]. Let us highlight, however, that they are valid beyond the RWA intrinsic to the standard Weisskopf-Wigner theory due to the polaronic variational methods. If one is interested in the reduced dynamics of the quantum emitters, as is usual in the spin-boson model or in the theory of quantum radiation, one can develop a Weisskopf-Wigner-type [30] theory by formally integrating the equations for the bosonic amplitudes and substituting them in the equations for the qubit amplitudes. Conversely, one may be interested in the scattering of propagating photons from the collection of quantum emitters, which would require the opposite process. In the following sections, we use both approaches, highlighting the importance of taking into account non-Markovian effects in the ultrastrong-coupling regime.

Let us note at this point that if the initial state contains some atomic coherences, the ansatz (14) must be generalized to include also an amplitude in the ground state $W_{\mathrm{sp}}\left[\alpha_{s}(t), \alpha_{k}(t)\right] \rightarrow W_{\mathrm{sp}}\left[\alpha_{s}(t), \alpha_{k}(t)\right]+\alpha_{\mathrm{g} . \mathrm{s} .}(t)$. However, this amplitude does not contribute with any term in the evaluation of Eq. (18) and thus $\alpha_{\text {g.s. }}(t)=\alpha_{\text {g.s. }}(0)$, whereas the time evolution of the remaining variational parameters is still described by Eq. (22). Yet including this ground-state amplitude may be necessary to calculate the dynamics of certain observables, such as the atomic coherences that are important for the coherent-incoherent transition of the spin-boson model.

At this stage, it is worth pointing out that our dynamical Ansatz (14) differs from the application of the so-called Davidov Ansatz, which arises in the study of exciton-phonon interactions, to the spin-boson model [47]. There are two crucial differences: (i) Our Ansatz is built in two steps, such that the polaron parameters are already fixed during the computation of the dynamical properties. This contrasts with the Davidov Ansatz, which consists of time-dependent polaron parameters with additional time-dependent variational weights for each spin state. (ii) Our Ansatz considers also additional single-photon excitations, which are absent in the Davidov Ansatz [47]. Property (i) will be crucial to be able to derive analytical expressions for the dynamics, whereas property (ii) will be crucial to describe the effect of spontaneous emission and photon scattering. 


\section{Matrix-product-state Ansatz}

The previous Ansätze will be compared with a wellestablished method [19] for the numerical simulation of the spin-boson model, which combines ideas from the quantum impurity Ansatz [48], matrix product operators [49], and Arnoldi-type evolution methods [50]. More precisely, we write down a variational wave function for the qubit-photon system as

$$
\left|\psi_{A}\right\rangle=\operatorname{tr}\left(A^{s_{1}} A^{n_{1}} \cdots A^{n_{M}}\right)\left|s_{1}, n_{1}, \ldots, n_{M}\right\rangle,
$$

where $s_{1}$ is the quantum state of the qubit, $n_{i}$ are the Fock states of $M$ different photon modes in frequency space, and the $A$ are different matrices with a size of up to $\chi^{2} \times n_{\max }$, where $\chi$ is the bond dimension of the MPS Ansatz and $n_{\max }$ is the maximum occupation of the bosonic modes. In addition to this encoding of the wave function, we efficiently write the spin-boson model as a long-range-interaction matrix product operator (MPO), which has a rather small bond dimension $O(3)$.

Combining the efficient representation of the Hamiltonian with the MPS wave function, we can either compute approximations to the ground state of spin-boson model or implement a time-evolution algorithm. In the first case we work by minimizing the energy functional

$$
E[\{A\}]=\frac{\left\langle\psi_{A}|H| \psi_{A}\right\rangle}{\left\langle\psi_{A} \mid \psi_{A}\right\rangle},
$$

with respect to the collection of numbers in all the matrices or tensors $A$. The minimization procedure is efficient due to the MPO representation of the Hamiltonian, even when it contains long-range interactions, and it is implemented with a generalization of the DMRG sweeping technique.

The time evolution is implemented using an approximation of the exponential of the Hamiltonian for short times. More precisely, we construct $\exp (-i H \Delta t)\left|\psi_{A}\right\rangle$ as a linear combination of MPSs $\sum_{n} c_{n}\left|\phi_{n}\right\rangle$, where the vectors $\left|\phi_{n}\right\rangle$ form a Krylov basis built with MPSs themselves, as explained in Ref. [50]. The use of Arnoldi expansions allows us to profit from the MPO expansion of the Hamiltonian and work with the long-range interactions, something that is much harder with Trotter expansions.

It has to be remarked that, while more accurate than the dynamical polaron Ansatz, the MPS method is more complex in terms of the implementation and computation. The number of parameters in an MPS variational wave-function scale as $N \times \chi^{2} n_{\text {max }}$, while in our few-photon dynamical Ansatz with one qubit, we have at most $(N+1) \times 2$ degrees of freedom. It is therefore interesting to use the MPS as a benchmark with which to assert the range of validity of the polaron Ansatz, with the idea of both having a flexible and simpler tool and also a way to implement potential analytical approximations and effective models.

\section{SINGLE-QUANTUM-EMITTER APPLICATIONS}

Once the different variational Ansatzë have been described, we will apply them to the simplest possible scenario: a single quantum emitter ultrastrongly coupled to a 1D em field. We will exploit the analytic polaron predictions based on the continuum model (4) to offer physical insight and benchmark the numerical polaron results based on the discretized model (3) with MPS simulations for an identical discretization. The main objective is to prove that the simple polaron Ansatz, in comparison to the more involved MPS technique, provides a sufficiently accurate description of both static and dynamical phenomena, with the hope that it will be established as a simple theoretical tool within the quantum optics community dealing with the ultrastrong-coupling regime.

\section{A. Static predictions}

\section{Continuum spin-boson model}

Let us consider the solution of the variational system of equations for the continuum single-impurity spin-boson model in Eqs. (1) and (4) [25-27]. For $N_{\mathrm{s}}=1$, the Ising Hamiltonian (11) reduces to a single-spin problem and the energy functional is

$$
\epsilon_{\mathrm{g} . \mathrm{s} .}^{\mathrm{P}}=\min _{\left[f_{1 k}\right]}\left\{J_{11}\left[f_{1 k}\right]-\frac{1}{2} \Delta_{\mathrm{r}}\left[f_{1 k}\right]\right\},
$$

where we have introduced the renormalized frequency

$$
\Delta_{\mathrm{r}}=\Delta_{1} e^{-\Xi_{1}\left[f_{i k}\right]} .
$$

The function minimization yields

$$
f_{1 k}=\frac{g_{1 k}}{\omega_{k}+\Delta_{\mathrm{r}}\left[f_{1 k}\right]},
$$

which amounts to a nonlinear system of equations for the polaron parameters. In this case, the variational spin parameters are

$$
c_{\sigma_{1}}=\delta_{\sigma_{1}, \downarrow} \theta\left(\Delta_{\mathrm{r}}\left[f_{1 k}\right]\right),
$$

where $\theta(x)$ is the Heaviside step function (i.e., if the renormalized qubit frequency vanishes, the variational ground state corresponds to the twofold degenerate Lang-Firsov transformed state). Therefore, by solving the system of implicit equations (27) and substituting in Eq. (28), we can recover the variational ground state (6) and calculate any observable. Let us note that by applying a nonvariational perturbative approach in the polaron-transformed picture, the same condition (27) has been found by imposing that the first-order perturbations vanish [51], as is customary in spin-wave approaches [52].

Note that the system of equations (27) can be rewritten in terms of a single implicit equation for the renormalized qubit frequency

$$
\Delta_{\mathrm{r}}^{\mathrm{CP}}=\Delta \exp \left\{-\int_{0}^{\infty} d \omega \frac{J(\omega)}{\pi\left(\omega+\Delta_{\mathrm{r}}\right)^{2}}\right\},
$$

where the superindex $\mathrm{CP}$ stands for the continuum polaron model and the exact spectral function corresponding to Eq. (4) is $J(\omega)=\pi \alpha \omega e^{-\omega / \omega_{\mathrm{c}}}$. This equation can be solved analytically in the so-called scaling limit $\omega_{\mathrm{c}} \gg \Delta \geqslant \Delta_{\mathrm{r}}$, where one finds

$$
\Delta_{\mathrm{r}}^{\mathrm{CP}}=\Delta\left(\frac{p \Delta}{\omega_{\mathrm{c}}}\right)^{\alpha /(1-\alpha)}
$$

with $p=e^{1+\gamma}$ and $\gamma$ as Euler's constant. Therefore, the polaron Ansatz predicts that the frequency of the quantum emitter gets renormalized as a consequence of its coupling to the photonic excitations (i.e., a photonic polaron cloud dresses the quantum emitter and leads to a renormalized transition 
frequency). This agrees with adiabatic renormalization-group arguments [20] and locates the localization-delocalization transition at $\alpha=1$, where the qubit frequency vanishes. This is an example of the so-called boundary quantum phase transitions that also arise in other condensed-matter contexts [28].

Using this result, we can recover the polaron (27) and spin (28) optimal parameters, which are be denoted by $\left[f_{1 k}^{\star}, c_{\sigma_{1}}^{\star}\right]$, and calculate any static observable by constructing the polaron ground state (6), such as

$$
\left\langle\sigma_{1}^{x}\right\rangle_{\mathrm{g} . \mathrm{C} .}^{\mathrm{CP}}=\left\langle\sigma_{1}^{y}\right\rangle_{\mathrm{g} . \mathrm{C} .}^{\mathrm{CP}}=0, \quad\left\langle\sigma_{1}^{z}\right\rangle_{\mathrm{g} . \mathrm{C} .}^{\mathrm{CP}}=-\frac{\Delta_{\mathrm{r}}^{\mathrm{CP}}}{\Delta} .
$$

For weak spin-boson interactions $\alpha \ll 1,\left\langle\sigma_{1}^{z}\right\rangle \rightarrow-1$, as expected from the ground state of a bare qubit $|\downarrow\rangle$. However, as the coupling to the bosonic bath is raised, the ground state starts populating also the excited bare state $|\uparrow\rangle$, until $\left\langle\sigma_{1}^{z}\right\rangle \rightarrow 0$ for $\alpha \rightarrow 1$. In the usual rotated basis of the spin-boson model [20], this corresponds to the localized phase.

\section{Discretized spin-boson model}

As emphasized previously, the continuum model serves to gain analytical insight into the static effects. However, it is the discrete version that can be benchmarked with MPS simulations and, more importantly, the model that provides a more accurate microscopic description of the bosonic bath in the physical transmission line. The solution of the variational problem in this discretized spin-boson model requires the use of numerics.

In the discretized model in Eqs. (1) and (3), the transmission line is divided into coupled segments such that the cutoff frequency depends on the input parameters $L$ and $N$, being $L$ the total physical length of the line and $N$ the number of segments of length $\delta x=L / N$ in which it is divided. We are interested in raising the number of segments $N$ for a fixed length $L$ such that the cutoff $\omega_{c}=v / \delta x$ can be maximized towards the above scaling limit and the linear region of the dispersion relation $\omega_{k} \sim v|k|$ around the qubit frequencies $\Delta_{i}$ contains as many photonic modes as possible. However, we also note that the computational complexity of the polaronMPS Ansatz grows with $N$ as the number of bosonic modes also increases $k_{n}=\frac{2 \pi}{L} n$, with $n \in\{0, \pm 1, \ldots, \pm N / 2\}$, and we cannot consider an arbitrarily large $N$.

In the discretized polaron Ansatz, we numerically diagonalize the transformed Hamiltonian (11) to obtain the state of minimum energy as a function of the variational parameters $\left[f_{1 k_{n}}, c_{\sigma_{1}}\right]$. Then we obtain the optimal parameters $\left[f_{1 k_{n}}^{\star}, c_{\sigma_{1}}^{\star}\right]$ by using a numerical optimization routine, minimizing this energy with respect to the variational parameters. Once these optimal parameters are obtained, we can calculate numerically parameters such as the renormalized qubit frequency, taken directly from the displaced Hamiltonian (11),

$$
\Delta_{\mathrm{r}}^{\mathrm{DP}}=\Delta \exp \left(-2 \sum_{n}\left|f_{1 k_{n}}^{\star}\right|^{2}\right)
$$

where the index DP stands for the discretized polaron.

In order to benchmark these static predictions with the MPS simulations, we should focus on some expectation values that can also be obtained through MPSs. An observable

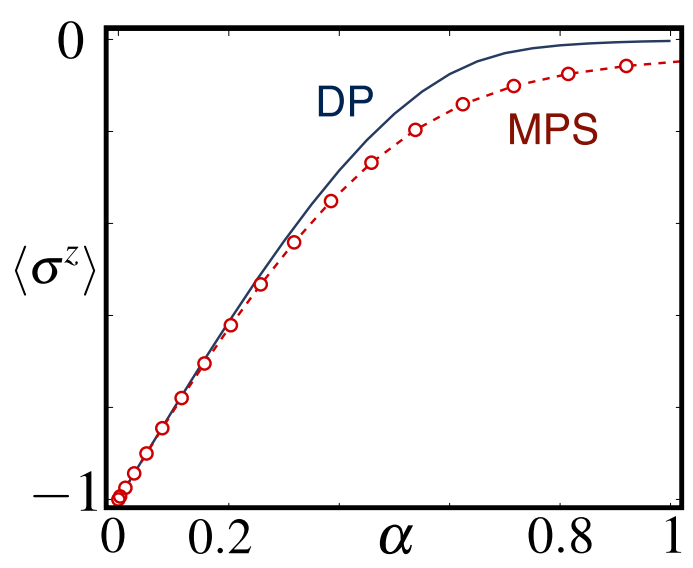

FIG. 1. Qubit polarization in the single-impurity spin-boson model: ground-state polarization $\left\langle\sigma_{1}^{z}\right\rangle_{\text {g.s. }}^{\eta}$ as a function of the spinboson coupling $\alpha=|g|^{2} / \pi v$. The dotted red line represents the value from MPS simulations $\left\langle\sigma_{1}^{z}\right\rangle_{\text {g.s. }}^{\text {MPS }}$ for $N=301$ and $L=10 \lambda_{0}$. The blue solid line represents $\left\langle\sigma_{1}^{z}\right\rangle_{\text {g.s. }}^{\mathrm{DP}}$ for the discrete Ansatz, with $L=10 \lambda_{0}$ and $N=301$.

of interest might be the qubit polarization, whose calculation is simple using the polaron ground-state $\left\langle\sigma_{1}^{z}\right\rangle_{\text {g.s. }}^{\text {DP }}=$ $\left\langle\psi_{\mathrm{s}}\left[c_{\sigma_{1}}^{\star}\right]\left|U_{\mathrm{P}}^{\dagger}\left[f_{1 k_{n}}^{\star}\right] \sigma_{1}^{z} U_{\mathrm{P}}\left[f_{1 k_{n}}^{\star}\right]\right| \psi_{\mathrm{s}}\left[c_{\sigma_{1}}^{\star}\right]\right\rangle$. After the numerical optimization, which yields $\left|\psi_{\mathrm{s}}\left[c_{\sigma_{1}}^{\star}\right]\right\rangle$ for the discretized spinboson model, we obtain the polarization by calculating

$$
\left\langle\left.\sigma_{1}^{z}\right|_{\mathrm{g} . \mathrm{s} .} ^{\mathrm{DP}}=\left\langle\psi_{\mathrm{s}}\left[c_{\sigma_{1}}^{\star}\right]\left|\sigma_{1}^{z}\right| \psi_{\mathrm{s}}\left[c_{\sigma_{1}}^{\star}\right]\right\rangle \exp \left(-2 \sum_{n}\left|f_{1 k_{n}}^{\star}\right|^{2}\right) .\right.
$$

Note that the eigenvectors are displaced by the polaron transformation (7), but so is the spin operator (hence the exponential due to the renormalization), so the result will be on the correct frame of reference. We represent these results in Fig. 1 with the same results from the MPS simulations $\left\langle\sigma_{1}^{z}\right\rangle_{\mathrm{g} . \mathrm{MS}}^{\mathrm{MPS}}$, where we observe reasonably good agreement between the polaron and MPS results for the same discretization with $N=301$ bosonic modes, which still lies far away from the scaling limit. We note that the discrete polaron results can be extended to a finer discretization with $N=601$ and approach the prediction of the continuum polaron (31) in the scaling limit. Such a high number of bosonic modes compromises the accuracy of the MPS simulation and highlights the ultimate power of the computationally less expensive polaron methods.

\section{B. Spontaneous emission}

So far, we have been concerned with the static properties of an ultrastrongly coupled qubit, although an even richer phenomenology arises out of equilibrium. In the context of the quantum theory of radiation, the typical situation is to study the evolution of an initially excited quantum emitter, whose population decays irreversibly as a consequence of the photonic reservoir. By looking at the coherences, one may find the analog of the coherent-incoherent dynamical crossover of the spin-boson model, where the spin displays a transition between damped oscillations and overdamped decay as the spin-boson coupling strength is increased beyond $\alpha=1 / 2$. The correct prediction of this dynamical effect is more 
challenging than the localization-delocalization transition and requires more involved techniques $[35,36]$ than the combination of the polaron static Ansatz with a Markovian master equation [27,41]. From previous nonvariational techniques [53], it becomes clear that the Markovian assumption must be abandoned if one wants to capture the correct dynamical behavior.

The objective of this section is to study the evolution of the populations and coherences of an initially excited quantum emitter, ultrastrongly coupled to the 1D em field by applying the dynamical Ansatz introduced previously, and we will show that the above previous limitations can be overcome with our method.

\section{Continuum spin-boson model}

For the single-impurity case $N_{\mathrm{s}}=1$, the situation simplifies as the Ising Hamiltonian (11) reduces to a single-spin problem and we have a simple ground state $\left|\psi_{\text {g.s. }}\right\rangle=|\downarrow\rangle$ together with a single-spin excitation $\left|\psi_{\mathrm{e}}^{1}\right\rangle=|\uparrow\rangle$, provided $\Delta_{\mathrm{r}}^{\mathrm{CP}}>0$. We can thus easily build the dynamical Ansatz (14) and obtain the corresponding differential equations (21), which are the variational analog of the Weisskopf-Wigner theory [30] of spontaneous emission

$$
\begin{aligned}
& i \partial_{t} \alpha_{1}(t)=\Delta_{\mathrm{r}}^{\mathrm{CP}} \alpha_{1}(t)+\sum_{k} \mathfrak{g}_{k 1} \alpha_{k}(t), \\
& i \partial_{t} \alpha_{k}(t)=\omega_{k} \alpha_{k}(t)+\mathfrak{g}_{k 1}^{*} \alpha_{1}(t)+\sum_{k^{\prime}} \mathfrak{f}_{k k^{\prime}}^{*} \alpha_{k^{\prime}}(t) .
\end{aligned}
$$

Here the couplings between the spin and photonic excitations arise,

$$
\mathfrak{g}_{k 1}=\frac{2 \Delta_{\mathrm{r}}^{\mathrm{CP}}}{\omega_{k}+\Delta_{\mathrm{r}}^{\mathrm{CP}}} g_{1 k},
$$

together with the additional couplings between the bare photonic modes

$$
\mathfrak{f}_{k k^{\prime}}=\frac{\Delta_{\mathrm{r}}^{\mathrm{CP}}}{\left(\omega_{k}+\Delta_{\mathrm{r}}^{\mathrm{CP}}\right)\left(\omega_{k^{\prime}}+\Delta_{\mathrm{r}}^{\mathrm{CP}}\right)}\left(g_{1 k} g_{1 k^{\prime}}^{*}+\text { c.c. }\right),
$$

which are symmetric $\mathfrak{f}_{k k^{\prime}}=\mathfrak{f}_{k^{\prime} k}$, as announced in the section introducing the dynamical Ansatz. Hence, this coupling matrix can be diagonalized by an orthogonal transformation leading to the general equations (22), which read in this case

$$
\begin{aligned}
& i \partial_{t} \alpha_{1}(t)=\Delta_{\mathrm{r}}^{\mathrm{CP}} \alpha_{1}(t)+\sum_{k} \tilde{\mathfrak{g}}_{k 1} \tilde{\alpha}_{k}(t), \\
& i \partial_{t} \tilde{\alpha}_{k}(t)=\left(\omega_{k}+\Delta \omega_{k}\right) \tilde{\alpha}_{k}(t)+\tilde{\mathfrak{g}}_{k 1}^{*} \alpha_{1}(t) .
\end{aligned}
$$

We integrate out the photonic modes by first changing to a rotating frame with $\alpha_{1}^{\prime}(t)=e^{i \Delta_{\mathrm{r}}^{\mathrm{CP}} t} \alpha_{1}(t)$ and $\tilde{\alpha}_{k}^{\prime}(t)=$ $e^{i\left(\omega_{k}+\Delta \omega_{k}\right) t} \tilde{\alpha}_{k}(t)$ and then substituting the expression

$$
\tilde{\alpha}_{k}^{\prime}(t)=-i \int_{0}^{t} d \tau \tilde{\mathfrak{g}}_{k 1}^{*} e^{i\left(\omega_{k}+\Delta \omega_{k}-\Delta_{\mathrm{r}}^{\mathrm{CP}}\right) \tau} \alpha_{1}^{\prime}(\tau),
$$

where we have assumed that there are no photonic excitations in the initially excited state, except for those intrinsic to the polaron cloud dressing the emitter. This leads to an integro- differential equation of the convolution type

$$
\partial_{t} \alpha_{1}^{\prime}(t)=-\int_{0}^{t} d \tau K_{1}(t-\tau) \alpha_{1}^{\prime}(\tau)
$$

where we have defined the memory kernel

$$
K_{1}(t)=\sum_{k}\left|\mathfrak{g}_{k 1}\right|^{2} e^{-i\left(\omega_{k}+\Delta \omega_{k}-\Delta_{\mathrm{r}}^{\mathrm{CP}}\right) t} .
$$

Since we are interested in deriving some analytical formulas, we need to replace all the sums by integrals over the spectral density. To do so, we note that the frequency shifts of the photons $\Delta \omega_{k}$ contribute at a higher order of the spin-boson coupling and neglecting them yields

$$
K_{1}(t)=\int_{0}^{\infty} \frac{J(\omega)}{2 \pi}\left(\frac{2 \Delta_{\mathrm{r}}^{\mathrm{CP}}}{\omega+\Delta_{\mathrm{r}}^{\mathrm{CP}}}\right) e^{-i\left(\omega-\Delta_{\mathrm{r}}^{\mathrm{CP}}\right) t} .
$$

(a) Markovian approximation. As is customary in the Weisskopf-Wigner theory [30], we perform a change of variables $\tau^{\prime}=t-\tau$ in the convolution (39) and a Markovian approximation to extend the integration domain to $\tau^{\prime} \in \mathbb{R}^{+}$ and substitute $\alpha_{1}^{\prime}(\tau) \rightarrow \alpha_{1}^{\prime}(t)$ in Eq. (39). In this case, after using $\int_{0}^{\infty} d \tau^{\prime} e^{-i \omega \tau^{\prime}}=\pi \delta(\omega)-i \mathcal{P}\left(\omega^{-1}\right)$, where $\mathcal{P}$ stands for Cauchy's principal value, the differential equation for the qubit can be expressed as

$$
i \partial_{t} \alpha_{1}^{\prime}(t)=\left(\delta_{1}-i \frac{\gamma_{1}}{2}\right) \alpha_{1}^{\prime}(t),
$$

where we have introduced the single-qubit decay rate $\gamma_{1}$ and the single-qubit Lamb shift $\delta_{1}$. The decay rate within this Markovian approximation can be easily evaluated

$$
\gamma_{1}=J\left(\Delta_{\mathrm{r}}^{\mathrm{CP}}\right)=\pi \alpha \Delta\left(\frac{p \Delta}{\omega_{\mathrm{c}}}\right)^{\alpha /(1-\alpha)} .
$$

This yields a very sensible result: The decay rate, which is given by the value of the spectral function evaluated at the bare qubit frequency $\gamma_{1}^{\mathrm{RWA}}=J(\Delta)$ within the usual RWA and weak-coupling assumptions [29], must be substituted by the value of the spectral function at the renormalized qubit frequency (30) according to Eq. (43). In agreement with more involved methods [20], our simple dynamical polaron Ansatz predicts that the localization-delocalization transition at $\alpha=$ 1 , where the renormalized qubit frequency vanishes, is also accompanied by a vanishing decay rate $\gamma_{1}=0$.

As a way to understand qualitatively this transition it is useful to use the picture of the polaron dressed states, which suggests that the atomic states get dressed by a cloud of photons that affect its polarization $\left\langle\sigma_{1}^{z}\right\rangle$ and its dynamical response. In the static regime and for sufficiently strong $\alpha$, the ground state is roughly a Schrödinger cat state and the polarization $\left\langle\sigma_{1}^{z}\right\rangle$ measures the coherence between the two states $|+\rangle,|-\rangle$, which gets degraded due to the vanishing overlap of the different photonic coherent states that are dressing each of these states. In the dynamical regime, the effect of this photonic cloud dressing the qubits is to "freeze" their dynamics, which is quantified by a renormalized transition frequency that tends to zero as the qubit-photon coupling is increased. As a consequence, the qubit would spontaneously emit photons at smaller frequencies, but the density of modes at those frequencies decreases for Ohmic environments (5). This is 
the qualitative reason that underlies the fact that the decay rate tends to zero as the localization-delocalization transition is crossed.

In this Markovian regime and in the scaling limit, the Lambtype shift can be calculated after solving the principal value integral and yields

$$
\delta_{1}=-\alpha \Delta_{\mathrm{r}}^{\mathrm{CP}}
$$

It is interesting to note that, in analogy to the original calculation of the Lamb shift of the em field where the self-energy is subtracted, the variational polaron formalism includes this self-energy directly in the ground state energy instead of the Lamb shift. This contrasts the calculations within the RWA [54], where the self-energy is not subtracted, and the Lamb shifts diverge with the cutoff frequency $\delta_{i}^{\mathrm{RWA}}=$ $-\alpha\left[\omega_{\mathrm{c}}-\frac{1}{2} \Delta \ln \left(\Delta / \omega_{\mathrm{c}}\right)\right]+\frac{\alpha}{2} \gamma \Delta$.

If we take the Lamb shift (44) together with the polaron renormalization, the frequency of the quantum emitter becomes $\Delta_{\mathrm{r}}^{\mathrm{CP}}(1-\alpha)$, which predicts that the evolution of coherences will stop at $\alpha=1$, instead of the prediction $\alpha=$ $1 / 2$ of the coherent-incoherent transition by other methods [20]. As we argue below, this is an artifact of the Markovian approximation and can be resolved by a more careful analysis.

(b) Non-Markovian approximation. Let us reconsider the integro-differential equation using resolvent-operator techniques $[29,55,56]$. By a Laplace transform and using the Bromwich contour to invert it, one can express the solution as $\alpha_{1}^{\prime}(t)=\frac{i}{2 \pi} \int_{-\infty}^{\infty} d \epsilon e^{-i \epsilon t} G(\epsilon)$, where we have introduced the propagator

$$
G(\epsilon)=\frac{1}{\left[\epsilon-\Delta_{\mathrm{r}}^{\mathrm{CP}}+\delta(\epsilon)\right]+i \frac{\gamma(\epsilon)}{2}}
$$

and we have defined the so-called level-shift operator

$$
\delta(\epsilon)=-\frac{2 \alpha\left(\Delta_{\mathrm{r}}^{\mathrm{CP}}\right)^{2}}{\Delta_{\mathrm{r}}^{\mathrm{CP}}+\epsilon},
$$

obtained from the principal value integral, and the so-called level broadening operator

$$
\gamma(\epsilon)=J(\epsilon)\left(\frac{2 \Delta_{\mathrm{r}}^{\mathrm{CP}}}{\epsilon+\Delta_{\mathrm{r}}^{\mathrm{CP}}}\right)^{2} .
$$

Note that, if one simply substitutes $\epsilon \rightarrow \Delta_{\mathrm{r}}^{\mathrm{CP}}$ in both operators, which is known as the single-pole approximation, one can easily perform the integral recovering the Markovian rate (43) and Lamb-type shift (44). However, if we are interested in the coherent-incoherent transition, a more careful analysis is required. In particular, by looking at solutions of $\epsilon-\Delta_{\mathrm{r}}^{\mathrm{CP}}+$ $\delta(\epsilon)=0$ where the propagator becomes maximal, one finds $\epsilon_{m}=\sqrt{1-2 \alpha} \Delta_{\mathrm{m}}$. A Taylor expansion about this solution shows that the propagator at $\alpha=1 / 2$ only leads to an exponential damping. Therefore, this non-Markovian treatment is capable of locating the coherent-incoherent transition at the correct spin-boson coupling strength $\alpha=1 / 2$.

\section{Discretized spin-boson model}

Let us now consider the dynamics under the discretized spin-boson model, which describes the spontaneous emission in the physical transmission line by using numerical means and can be benchmarked again with MPS simulations, serving thus as a test of the validity of our method. Regarding the dynamical polaron Ansatz with such a discretized model, a clear advantage is that the vector of time-dependent variational parameters becomes finite $\boldsymbol{\alpha}(t)=\left[\alpha_{1}(t), \alpha_{k_{1}}(t), \alpha_{k_{2}}(t), \ldots, \alpha_{k_{N}}(t)\right]^{t}$ and one can directly solve the Schrödinger equation $i \partial_{t} \boldsymbol{\alpha}=\mathbb{H}_{\mathrm{P}} \boldsymbol{\alpha}$ without making any connections to the Weisskopf-Wigner typical approximations. From a numerical perspective, we can extend this Ansatz, at the same computational effort, to consider also the amplitudes of the ground state in the presence or absence of one additional photonic excitation for the different modes. We thus obtain a $2(N+1) \times 2(N+1)$ matrix $\mathbb{H}_{\mathrm{P}}$ by evaluating numerically Eq. (18) for the different matrix elements. Then the system of differential equations is solved numerically after specifying a particular initial condition, such as $\alpha_{1}(0)=1$.

In order to study the spontaneous emission rate, we know that the probability amplitude of the qubit in the excited state $\alpha_{1}(t)$ oscillates with frequency $\Delta_{\mathrm{r}}+\delta_{1}$ and decays with $\gamma_{1} / 2$. If we consider the expectation value of the excitation number operator $\sigma_{1}^{+} \sigma_{1}^{-}$, we are able to directly extract the spontaneous emission rate via exponential fitting of

$$
\boldsymbol{\alpha}(t)^{\dagger}\left(\sigma_{1}^{+} \sigma_{1}^{-}\right) \boldsymbol{\alpha}(t)=\left|\alpha_{1}(t)\right|^{2}=e^{-\gamma_{1} t},
$$

which we plot as a function of the coupling strength in Fig. 2, along with the same quantity obtained with the MPS Ansatz. The agreement between both approaches is remarkable and serves as a test of the validity of the proposed dynamical polaron Ansatz. Let us remark that, by solving directly the variational Schrödinger equation, no Markovian approximations are taken with the discretized Ansatz and it should thus give more accurate predictions than the continuum results based on this assumption.

Although we have used a general exponential approximation (48) for the decay of the initial state with our Ansatz, for large values of the qubit-photon coupling $\alpha$, the evolution of the excited-state population can actually be decomposed in two terms: The first one is a fast prethermalization, which can be understood from the decoherence of the macroscopic superposition of the atomic states $|+\rangle,|-\rangle$ present in the initial

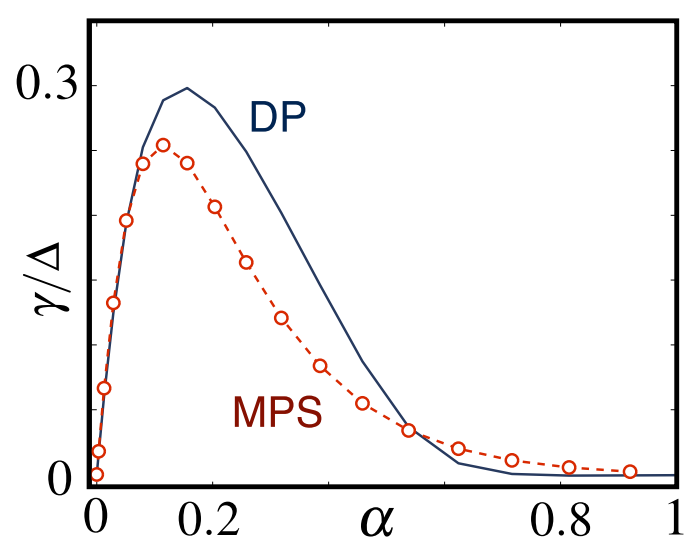

FIG. 2. Spontaneous emission rate. The blue solid line represents the calculated with the discrete polaron Ansatz for $N=301$ and $L=10 \lambda_{0}$. The red dotted line represents the MPS data for the same input parameters. 


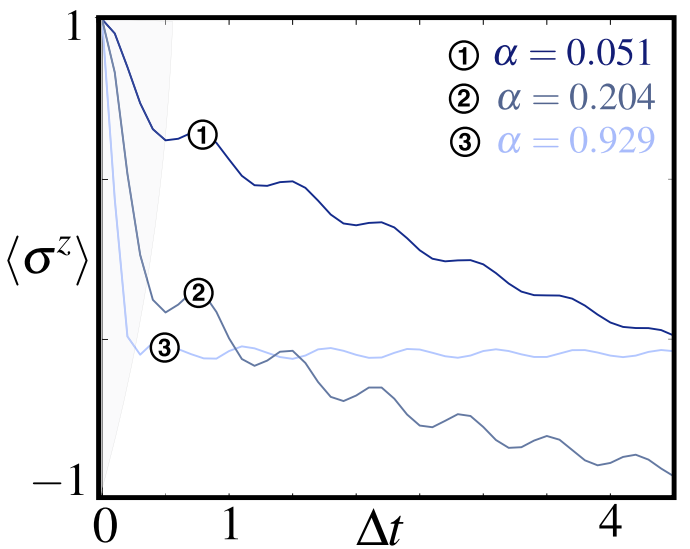

FIG. 3. Different polarization decay regimes: dynamics of the polarization of the qubit in the MPS numerics for three different values of the spin-boson couplings $\alpha \in\{0.051,0.204,0.929\}$. Two regimes of evolution can be distinguished: For small times there is a fast cutoff-dependent prethermalization depicted by a shaded region, followed by an exponentially damped regime due to photon emission.

state $|\psi(0)\rangle=|\uparrow\rangle \otimes|0\rangle=\frac{1}{\sqrt{2}}(|+\rangle+|-\rangle) \otimes|0\rangle$. In the limit $\alpha \rightarrow \infty$, this initial state evolves into a Schrödinger cat state where each of the $|+\rangle,|-\rangle$ is dressed by a photonic coherent state. In this regime, the excited-state probability can be shown to decay algebraically

$$
P(t)=\frac{1}{1+\left(\omega_{\mathrm{c}} t\right)^{2}},
$$

which is an exact result for the Ohmic spin-boson model at $\alpha \rightarrow \infty$ [57]. For finite $\alpha$, in addition to the previous fast decay, the excited-state probability can decay due to the emission of real photons onto the environment. This leads to an exponential decay with a rate that can be approximated by our Ansatz in Eq. (43) and is displayed in Fig. 2.

In the scaling limit, where the cutoff is sent to $\omega_{\mathrm{c}} \rightarrow \infty$, the algebraic decay (49) is almost instantaneous. The dynamical polaron Ansatz cannot account for such small time scales, as it is limited to $t \gg 1 / \omega_{\mathrm{c}}$, and models this decay as an instantaneous process encoded in the polaron unitary (7). Conversely, the MPS techniques can in fact account for this very fast transient state, where the decay is not exponential, as shown in Fig. 3. Therefore, to extract the exponential decay (48) from the MPS numerics, one has to consider a time window $\left[t_{1}, t_{2}\right]$, where $t_{1}$ is sufficiently large $t_{1} \gg 1 / \omega_{\mathrm{c}}$ such that the above transient where Eq. (49) plays a role is not included in the fit.

An important and crucial advantage of the discretized model is that, for the same effort, one can get information about the dynamics of the photons. As emphasized previously, this is rather unique to our method and very important in light of the current experiments. For instance, it may be of interest to study the photon number density $\left\langle a_{k}^{\dagger} a_{k}\right\rangle$ after the qubit has relaxed completely. It will give an indirect measure of the qubit frequency akin to a spectroscopy experiment, since the emitted photon is expected to have the same energy as the qubit excitation.
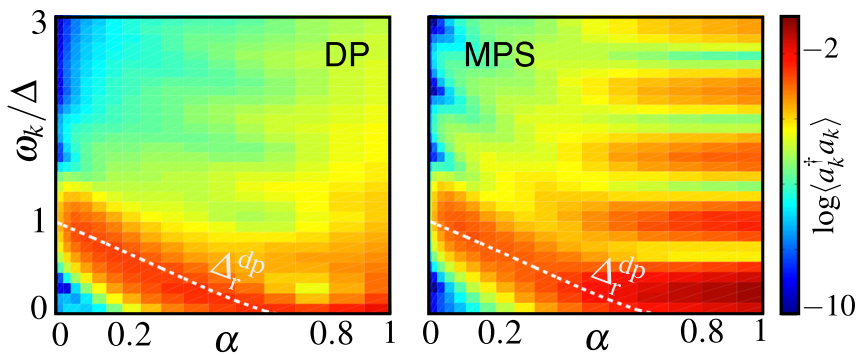

FIG. 4. Photon density in the spontaneous emission for the discrete polaron Ansatz (left) and the MPS Ansatz (right). The $z$ axis in these plots has a logarithmic scale (with base 10) and so does the common color bar. The white dashed line shows the renormalized qubit frequency over the surface plots, confirming visually that the bosonic resonances coincide with it. Both models display oscillations in $\omega_{k}$ caused by the boundary conditions and the nonequilibrium initial condition, which gives two possible field states that interfere with each other. Having different initial states in each model, these oscillations appear far more noticeable in the MPS simulations, but they also occur in the discrete polaron ones.

We have calculated the photon distribution as a function of the coupling strength and the frequency of the modes within our discrete polaron Ansatz and compared the predictions to another simulation with the same parameters using the MPS Ansatz. As shown in Fig. 4, both methods show clearly a peak in the distribution around the renormalized qubit frequency calculated through the static Ansatz in the previous section and thus confirm the above intuition that this photonic observable serves as a spectroscopy probe. Let us note that, since we are using periodic boundary conditions for the transmission line, time must be long enough for the spin excitation to have decayed, but sufficiently short that the photon cannot get around the line and scatter with the emitter again (revival time).

\section{Single-photon scattering}

The other type of experiment that can be simulated with these tools is the scattering of one photon traveling in the transmission line with one qubit impurity. The photon distribution, after the collision has occurred, will give information about the transmission and reflection coefficients of the photons in the transmission line ( $\mathfrak{T}$ and $\mathfrak{R}$, respectively).

In the polaron-transformed frame, we can consider the initial state as a product of its two components, the qubit in the ground state and the photon distribution specified by the probability amplitudes $\alpha_{k_{n}}(0)$, namely,

$$
\left|\psi_{\mathrm{exc}}(0)\right\rangle=\sum_{n} \alpha_{k_{n}}(0) a_{k_{n}}^{\dagger}|0\rangle \otimes\left|\psi_{\text {g.s. }}\right\rangle .
$$

During the collision and some time after it has happened, the photon and qubit interact such that the wave function is no longer in a product state. However, for long-enough times, the qubit will have decayed completely and we can describe the state again as

$$
\left|\psi_{\mathrm{exc}}(\infty)\right\rangle=\sum_{n} \alpha_{k_{n}}(\infty) a_{k_{n}}^{\dagger}|0\rangle \otimes\left|\psi_{\text {g.s. }}\right\rangle,
$$

with a different photon distribution specified by the probability amplitudes $\alpha_{k_{n}}(\infty)$. This enables us to define the transmission 

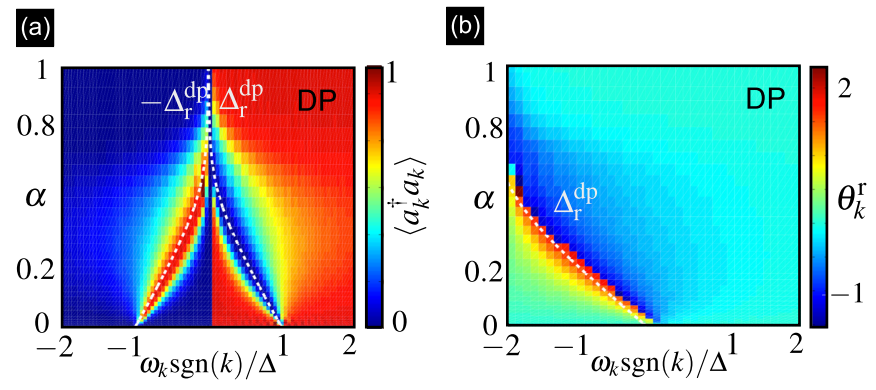

FIG. 5. Normalized photon density in the single-photon scattering. (a) The horizontal axis does not display the frequency $\omega$ but the product of $\omega$ and the sign of the momentum, to tell apart modes with the same energy but opposite traveling direction. White lines represent the renormalized photon frequency, which coincides with the resonances as expected. The right side of the graph corresponds to the norm of the transmission coefficient $|\mathfrak{T}|^{2}$ and the left side to the norm of the reflection coefficient $|\Re|^{2}$. (b) Phase shift of the reflection coefficient in the single-photon scattering simulation.

and reflection coefficients as

$$
\begin{aligned}
& \mathfrak{r}_{k_{n}}=\frac{\left|\alpha_{-k_{n}}(\infty)\right|}{\left|\alpha_{+k_{n}}(0)\right|} e^{i \theta_{k_{n}}^{\mathrm{r}}} e^{i \omega_{k_{n}} t}, \\
& \mathfrak{t}_{k_{n}}=\frac{\left|\alpha_{+k_{n}}(\infty)\right|}{\left|\alpha_{+k_{n}}(0)\right|} e^{i \theta_{k_{n}}^{\mathrm{t}}} e^{i \omega_{k_{n}} t},
\end{aligned}
$$

where $\theta_{k_{n}}^{\mathrm{t}}$ and $\theta_{k_{n}}^{\mathrm{r}}$ are the complex phases of the coefficients and the $e^{i \omega_{k_{n}} t}$ include all the phases due to the evolution. The calculation of transmission and reflection coefficients $\mathfrak{T}_{k_{n}}=$ $\left|\mathfrak{t}_{k_{n}}\right|^{2}$ and $\Re_{k_{n}}=\left|\mathfrak{r}_{k_{n}}\right|^{2}$, respectively, can be achieved from the expectation value of the photon number operator.

Instead of having an initially excited qubit, this time we initialize the system with one single photon traveling in one direction of the line with the qubit in its ground state, which corresponds to Eq. (50) for a particular set of probability amplitudes. This photon will be very localized and will have a flat frequency distribution, but only in the positive momentum. After the scattering, some of the modes will be absorbed by the qubit and reemitted afterward in both directions, effectively being reflected or transmitted from the qubit. This will be depicted by modes with positive momentum vanishing and some other modes with the same energy but opposite momentum appearing. To check that our polaron predictions for this scattering experiment are consistent, we compare the photon density resonances with the renormalized qubit gap obtained through the static Ansatz. We can confirm this in Fig. 5, which also shows that for high enough couplings, the qubit stops interacting with the photons because its dynamics gets frozen.

This analysis of the probability density of the photons gives the norm of the transmission and reflection coefficients. Additionally, we can also calculate the phase shift as the difference between the final and initial phases, by subtracting the phase due to the time evolution. To perform this task, we run two simulations, the first one with the qubit connected to the line and the second one without it (equivalent to setting the coupling strength to zero). We then extract the phase shift of each mode as the division between the amplitude of that mode in the scattered and in the free case as in Eq. (52). Figure 5(b) shows this scattering phase for different values of the coupling strength. As expected, the phase jump occurs around the renormalized qubit frequency.

\section{CONCLUSION AND OUTLOOK}

In this work we have introduced a simple and general technique based on a time-dependent variational principle to describe dynamical aspects of a system of quantum emitters ultrastrongly coupled to the 1D em field beyond the RWA regime. The dynamics of the system is described within a region of the Hilbert space that is relevant for the typical experimental situations encountered in spontaneous emission of initially excited emitters or scattering of an incoming photon wave packet by the quantum emitters. By a physically motivated parametrization of this relevant region of the Hilbert space, these dynamical variational Ansätze become computationally less expensive than matrix-product-state simulations and allow us to develop some physical insight in certain regimes where analytical results can be derived. More importantly, they allow us to address the dynamics of the photonic degrees of freedom, which becomes very relevant in light of the recent experimental progress.

In order to benchmark the accuracy of our variational techniques, we have performed a detailed comparison of static and dynamical predictions for a single quantum emitter ultrastrongly coupled to the em field. In this context, we have compared the predictions of these simple polaron methods with the numerical results of the quasiexact MPS methods for both equilibrium and nonequilibrium scenarios, showing remarkable agreement in typical situations of spontaneous emission and photon scattering. Given the computational simplicity of the introduced Ansatz, as compared to the complexity of MPS methods, and its demonstrated reasonable accuracy, we believe that it can become a useful theoretical tool for the quantum optics community interested in the ultrastrong-coupling regime.

The computational advantage of the introduced Ansatz becomes even more important as the number of quantum emitters is increased. Although we have focused in the present paper on single-emitter applications, the general scheme presented in Sec. III can be applied to any number of emitters. For instance, we can start from the static polaron Ansatz for a couple of quantum emitters $N_{\mathrm{s}}=2$ [43] and build our dynamical Ansatz to analyze photon-mediated interactions and collective effects in the spontaneous emission. To illustrate some of the power of our dynamical Ansatz, let us advance some of the predictions that will be detailed elsewhere.

\section{Outlook for two-emitter applications}

The static variational problem for two identical emitters can be reduced to a couple of implicit equations, one of the renormalized qubit frequencies $\Delta_{\mathrm{r}}$ and another one for the photon-mediated Ising interactions $J_{\mathrm{I}}$, which display an interesting dependence on the interqubit distance [43]. Let us discuss two particularly simple limits. (a) At large distances, 
the interactions are vanishingly small, such that

$$
J_{\mathrm{I}} \rightarrow 0, \quad \Delta_{\mathrm{r}}=\Delta\left(p_{\infty} \frac{\Delta}{\omega_{\mathrm{c}}}\right)^{\alpha /(1-\alpha)},
$$

where $p_{\infty}=p$ coincides with the single-emitter predictions (30). We thus see that for $\alpha=1$, the frequencies of both qubits renormalize to zero in the scaling limit, such that the localization-delocalization transition of both impurities occurs at the same critical coupling as the single-impurity Ohmic spin-boson model (30). (b) Conversely, at short distances $d \ll v / \omega_{\mathrm{c}}$, the interactions are ferromagnetic and increase with the cutoff

$$
J_{\mathrm{I}} \rightarrow-\alpha \omega_{c}, \quad \Delta_{\mathrm{r}}=\Delta\left(p_{0} \frac{\Delta}{\omega_{\mathrm{c}}}\right)^{2 \alpha /(1-2 \alpha)},
$$

where $p_{0}=(p / \alpha)^{1 / 2}$, provided $\alpha \omega_{\mathrm{c}} / \Delta \gg 1$. In this case, the renormalized frequency vanishes at a smaller spin-boson coupling $\alpha=\frac{1}{2}$, which was used in [43] to locate the localization-delocalization phase transition.

Turning our attention to the dynamical effects, let us note that the qubits can also exchange real photons beyond the virtual photons associated with the polaron clouds and this leads to collective effects in the two-impurity spin-boson model. In order to account for these effects, we note that the variational spin Hamiltonian (11) evaluated at $\left[f_{i k}^{\star}\right]$ leads to two spin-wave excitations that can be excited from the ground state if the spins absorb a real photon from the em environment. In the Markovian limit of the Weisskopf-Wigner-type theory analogous to the single-emitter case (42), we find that the amplitudes of the excitations fulfill

$$
\begin{aligned}
& i \partial_{t} \alpha_{1}^{\prime}(t)=\left(\delta_{1}-i \frac{\gamma_{1}}{2}\right) \alpha_{1}^{\prime}(t)+\left(g_{12}-i \frac{\gamma_{12}}{2}\right) \alpha_{2}^{\prime}(t), \\
& i \partial_{t} \alpha_{2}^{\prime}(t)=\left(\delta_{2}-i \frac{\gamma_{2}}{2}\right) \alpha_{2}^{\prime}(t)+\left(g_{21}-i \frac{\gamma_{21}}{2}\right) \alpha_{1}^{\prime}(t),
\end{aligned}
$$

where we have introduced the single-qubit $\gamma_{i}$ and collective relaxation rates $\gamma_{12}=\gamma_{21}$ as well as the single-qubit $\delta_{i}$ Lamb shifts and collective interactions $g_{12}=g_{21}$. Let us comment on the particular expressions for these parameters in order.

\section{Spontaneous decay rates}

The incoherent spontaneous emission is given by the decay rates

$$
\gamma_{i}=J\left(\Delta_{\mathrm{r}} \zeta\right) \chi^{2}, \quad \gamma_{12}=\gamma_{1} \cos \left(\frac{\Delta_{\mathrm{r}} \zeta}{v} d\right),
$$

where we introduced $\zeta=\left(1+J_{\mathrm{I}}^{2} / \Delta_{\mathrm{r}}^{2}\right)^{1 / 2}, \chi=\zeta \eta /\left(1+\zeta^{2}\right)$, and $\eta=\left\{\sqrt{\zeta+1}\left(\zeta^{-1}+1\right)+\sqrt{\zeta-1}\left(\zeta^{-1}-1\right)\right\} / \sqrt{2 \zeta}$.

These expressions allow us to study how the spontaneous emission of the pair of qubits gets modified as the coupling to the em field increases, eventually entering the ultrastrongcoupling regime. Regarding the individual decay rates, their change with respect to the weak-coupling value is caused by both the renormalization of the qubit frequency $\Delta_{\mathrm{r}}<\Delta$ and the Ising interactions due to virtual photon exchange $\zeta>1$. As a consequence, the individual decay rates will also depend on the interqubit distance. In the scaling limit $\Delta \ll \omega_{\mathrm{c}}$, using the results form the previous section (53) and (54), we find

$$
\gamma_{i}= \begin{cases}\pi \alpha \Delta\left(p_{\infty} \frac{\Delta}{\omega_{c}}\right)^{\alpha /(1-\alpha)}, & d \rightarrow \infty \\ \pi \alpha \chi_{0}^{2} \zeta_{0} \Delta\left(p_{0} \frac{\Delta}{\omega_{c}}\right)^{2 \alpha /(1-2 \alpha)}, & d \rightarrow 0,\end{cases}
$$

where we have introduced $\zeta_{0}=\left[1+\left(\alpha \omega_{\mathrm{c}} / \Delta_{\mathrm{r}}\right)^{2}\right]^{1 / 2}$ and $\chi_{0}, \eta_{0}$ are the above parameters evaluated at $\zeta=\zeta_{0}$.

Let us first comment on the regime where the qubits are so far apart that the Ising interaction is negligible. When the spin-boson coupling is sufficiently weak $\alpha \ll 1$, we recover the result expected from the usual Weisskopf-Wigner theory of spontaneous emission $\gamma_{i}=J(\Delta)=\pi \alpha \Delta$, which rests on the RWA. Regarding the collective spontaneous emission in this weak-coupling regime, we observe that it gets suppressed for distances $d=v(2 n+1) \pi / 2 \Delta$ with $n \in \mathbb{Z}^{+}$, which coincides again with the predictions based on the RWA [54,58,59]. In contrast, the collective spontaneous emission attains a maximum at $d=v n \pi / \Delta$ with $n \in \mathbb{Z}^{+}$, leading to subradiant (superradiant) channels related to the singlet (triplet) Bell states being dark states [58].

These predictions are modified as the spin-boson coupling is raised or as the qubits get closer. By raising the spinphoton couplings, still at large distances, we see that the individual emission rates depend on the spectral density evaluated at the renormalized qubit frequencies $\gamma_{i}=J\left(\Delta_{\mathrm{r}}\right)=$ $\pi \alpha \Delta\left(p_{\infty} \Delta / \omega_{c}\right)^{\alpha /(1-\alpha)}$. Therefore, the individual and collective spontaneous emissions get totally suppressed in the localized regime $\alpha=\alpha_{\mathrm{c}}^{\infty}=1$ at sufficiently large distances. In the delocalized regime $\alpha<\alpha_{\mathrm{c}}^{\infty}=1$, both individual and collective incoherent decay channels contribute to the dynamics. In contrast to the RWA, the collective rates get suppressed (enhanced) at distances that are also controlled by the renormalized frequency $d=v(2 n+1) \pi / 2 \Delta_{\mathrm{r}}(d=$ $v n \pi / 2 \Delta_{\mathrm{r}}$ ) with $n \in \mathbb{Z}^{+}$. Therefore, in order to exploit such collective decay to engineer entangled states dissipatively, as proposed in [58,59], it is very important to estimate the correct distance dependence by a careful calculation of the renormalized frequency.

Let us now move onto the other regime where the standard Weisskopf-Wigner predictions are modified: short interqubit distances. Even in the weak-coupling regime where $\Delta_{\mathrm{r}} \approx \Delta$, the strength of the individual decay rates $\gamma_{i}=J\left(\Delta \zeta_{0}\right) \chi_{0}^{2}=$ $\pi \alpha \Delta\left(\chi_{0}^{2} \zeta_{0}\right)$ is different from the RWA predictions $\gamma_{i}=$ $J(\Delta)=\pi \alpha \Delta$ as a consequence of the ferromagnetic Ising interactions $\chi_{0}^{2} \zeta_{0} \neq 1$. The distance where the collective decay rates get suppressed (enhanced) is also modified by the presence of interactions $d=v(2 n+1) \pi / 2 \Delta \zeta_{0}\left(d=v n \pi / 2 \Delta \zeta_{0}\right)$ with $n \in \mathbb{Z}^{+}$. Hence, in order to exploit these collective decays to engineer entangled states dissipatively, it is also important to estimate the correct distance by a careful calculation of the interactions due to virtual photon exchange. The differences with respect to the standard Weisskopf-Wigner theory become more important as the spin-boson coupling is increased. As shown in Eqs. (57) and (56), the individual and collective spontaneous emissions are suppressed in the localized regime, which at short distances occurs for a weaker coupling $\alpha=$ $\alpha_{\mathrm{c}}^{0}=1 / 2$. 


\section{Lamb shifts and photon-mediated interactions}

In addition to the above incoherent decay rates, the qubits will also suffer a frequency shift and a coherent interaction due to the exchange of real photons (i.e., on-shell contribution). The frequency shifts (i.e., Lamb shifts) of the qubits due to the photonic environment can be expressed as

$$
\delta_{1}=\delta_{2}=-\alpha \frac{\eta \chi}{2} \Delta_{\mathrm{r}}\left[1-f_{\mathrm{L}}\left(\frac{\Delta_{\mathrm{r}}}{\omega_{\mathrm{c}} \zeta}\right)\right]
$$

where $f_{\mathrm{L}}(x)=\frac{\zeta^{2}}{1+\zeta^{2}} \operatorname{Re}\left\{e^{x} E_{1}(x)-e^{-x \zeta^{2}} E_{1}\left(-x \zeta^{2}\right)\right\}$ is defined in terms of the exponential integral $E_{1}(z)=\int_{z}^{\infty} d t e^{-t} / t$. In the scaling limit and according to Eqs. (53) and (54), the Lamb shifts become

$$
\delta_{i}= \begin{cases}-\alpha \Delta\left(p_{\infty} \frac{\Delta}{\omega_{c}}\right)^{\alpha /(1-\alpha)}, & d \rightarrow \infty \\ -\alpha \frac{\eta_{0} \chi_{0}}{2}\left(1-\frac{\zeta_{0}^{2} \log \zeta_{0}^{2}}{1+\zeta_{0}^{2}}\right) \Delta\left(p_{0} \frac{\Delta}{\omega_{c}}\right)^{2 \alpha /(1-2 \alpha)}, & d \rightarrow 0,\end{cases}
$$

As occurs for the single-emitter case, a calculation beyond the Markovian approximation should be performed to locate exactly the coherent-incoherent transition.

Let us now focus on the coherent photon-mediated interactions $g_{12}$, which have two contributions. As argued in the previous section, $J_{\mathrm{I}}$ is caused by the exchange of virtual off-shell photons. The remaining contribution corresponds to the exchange of real on-shell photons. In particular, again in the scaling limit, we find

$$
g_{12}=J_{\mathrm{I}}+\frac{\pi}{2} \zeta \chi^{2} \alpha \Delta_{\mathrm{r}} \sin \left(\frac{\Delta_{\mathrm{r}} \zeta}{v} d\right)+\delta g_{12}\left(i \frac{\Delta_{\mathrm{r}}}{v \zeta} d+\frac{\Delta_{\mathrm{r}}}{\zeta \omega_{\mathrm{c}}}\right),
$$

where we have introduced

$$
\delta g_{12}(z)=\frac{-\chi^{2} \alpha \Delta_{\mathrm{r}}}{2}\left\{1+\operatorname{Im} z f_{\mathrm{I}}(z)-\zeta\left[f_{\mathrm{R}}(z)-f_{\mathrm{R}}\left(-z^{*} \zeta^{2}\right)\right]\right\}
$$

and we have used the functions

$$
f_{\mathrm{I}}(z)=\operatorname{Im}\left\{E_{1}(z) e^{z}\right\}, \quad f_{\mathrm{R}}(z)=\operatorname{Re}\left\{E_{1}(z) e^{z}\right\}
$$

At large distances, where the Ising contribution $J_{\mathrm{I}}$ due to virtual photon exchange vanishes (53), the qubit-qubit interaction is due to the exchange of real photons. Moreover, $\delta g_{12}$ also vanishes at large distances and we obtain

$$
g_{12}=\frac{\pi}{2} \alpha \Delta_{\mathrm{r}} \sin \left(\frac{\Delta_{\mathrm{r}}}{v} d\right)
$$

Let us first consider the weak-coupling limit $\alpha \ll 1$, where the qubit frequencies approach the bare value $\Delta_{\mathrm{r}} \approx \Delta$. In this case, we obtain $g_{12}=\frac{\pi}{2} \alpha \Delta \sin \left(\frac{\Delta}{v} d\right)$, in accord with the results based on the RWA [54]. We thus recover the result that the photon-exchange interactions are suppressed for the distances $d=v n \pi \Delta$ with $n \in \mathbb{Z}^{+}$, where the collective spontaneous decay (56) is maximal, and vice versa [58]. As the spin-boson coupling is increased, these distances are changed as a consequence of the renormalization of the qubits frequency $d=v n \pi \Delta_{\mathrm{r}}$ with $n \in \mathbb{Z}^{+}$. However, when the spin-photon coupling is sufficiently large, the interactions are totally suppressed since

$$
\left|g_{12}\right| \leqslant \frac{\pi}{2} \alpha \Delta\left(p_{\infty} \frac{\Delta}{\omega_{c}}\right)^{\alpha /(1-\alpha)} \rightarrow 0, \quad \alpha \rightarrow \alpha_{\mathrm{c}}^{\infty}=1
$$

and no coherent swap of the excitation can occur. We thus see that in the localized phase, all coherent and incoherent processes are inhibited.

The situation changes considerably at very short distances, where the coherent Ising part $J_{\text {I }}$ given by Eq. (54) becomes the leading term in the scaling limit. In this case, the interactions

$$
g_{12}=-\alpha \omega_{\mathrm{c}}-\frac{1}{2} \alpha \chi_{0}^{2} \Delta_{\mathrm{r}} \approx-\alpha \omega_{\mathrm{c}}
$$

become independent of the renormalized qubit frequency and diverge with the cutoff frequency. Such behavior is consistent with the results based on the RWA and predicts that the excitation can always be coherently swapped between the qubits, provided they are close enough. In the localized regime $\alpha \geqslant \alpha_{\mathrm{c}}^{0}=\frac{1}{2}$, the decay channels are suppressed such that the qubits continue swapping the excitation indefinitely.

Let us finally highlight that, just as the simple Markovian approximation leading to Eqs. (55) can be improved to predict the coherent-incoherent transition by taking into account non-Markovian effects, it can also be improved by taking into account retardation times for the exchange of photons [60], thus making an interesting connection with the emerging causality for spin-boson models discretized on a lattice [61].

\section{ACKNOWLEDGMENTS}

The authors acknowledge support from Spanish Mineco Project No. FIS2012-33022, CAM Research Network QUITEMAD+, and EU FP7 FET Open project PROMISCE.
[1] O. Astafiev, A. M. Zagoskin, A. A. Abdumalikov, Y. A. Pashkin, T. Yamamoto, K. Inomata, Y. Nakamura, and J. S. Tsai, Science 327, 840 (2010).

[2] P. Lodahl, S. Mahmoodian, and S. Stobbe, Rev. Mod. Phys. 87, 347 (2015).

[3] D. E. Chang, A. S. Sørensen, E. A. Demler, and M. D. Lukin, Nat. Phys. 3, 807 (2007).

[4] I.-C. Hoi, C. M. Wilson, G. Johansson, T. Palomaki, B. Peropadre, and P. Delsing, Phys. Rev. Lett. 107, 073601 (2011).
[5] I.-C. Hoi, T. Palomaki, J. Lindkvist, G. Johansson, P. Delsing, and C. M. Wilson, Phys. Rev. Lett. 108, 263601 (2012).

[6] C. Eichler, C. Lang, J. M. Fink, J. Govenius, S. Filipp, and A. Wallraff, Phys. Rev. Lett. 109, 240501 (2012).

[7] A. F. van Loo, A. Fedorov, K. Lalumière, B. C. Sanders, A. Blais, and A. Wallraff, Science 342, 1494 (2013).

[8] K. Lalumière, B. C. Sanders, A. F. van Loo, A. Fedorov, A. Wallraff, and A. Blais, Phys. Rev. A 88, 043806 (2013).

[9] J. Shen and S. Fan, Opt. Lett. 30, 2001 (2005). 
[10] J.-T. Shen and S. Fan, Phys. Rev. Lett. 95, 213001 (2005).

[11] T. Shi, S. Fan, and C. P. Sun, Phys. Rev. A 84, 063803 (2011).

[12] B. Peropadre, J. Lindkvist, I.-C. Hoi, C. M. Wilson, J. J. GarcíaRipoll, P. Delsing, and G. Johansson, New J. Phys. 15, 035009 (2013).

[13] T. Shi and C. P. Sun, Phys. Rev. B 79, 205111 (2009).

[14] M. Laakso and M. Pletyukhov, Phys. Rev. Lett. 113, 183601 (2014).

[15] T. Shi, D. E. Chang, and J. I. Cirac, Phys. Rev. A 92, 053834 (2015).

[16] T. Niemczyk, F. Deppe, H. Huebl, E. P. Menzel, F. Hocke, M. J. Schwarz, J. J. Garcia-Ripoll, D. Zueco, T. Hümmer, E. Solano, A. Marx, and R. Gross, Nat. Phys. 6, 772 (2010).

[17] P. Forn-Díaz, J. Lisenfeld, D. Marcos, J. J. García-Ripoll, E. Solano, C. J. P. M. Harmans, and J. E. Mooij, Phys. Rev. Lett. 105, 237001 (2010).

[18] J. Bourassa, J. M. Gambetta, A. A. Abdumalikov, Jr., O. Astafiev, Y. Nakamura, and A. Blais, Phys. Rev. A 80, 032109 (2009).

[19] B. Peropadre, D. Zueco, D. Porras, and J. J. García-Ripoll, Phys. Rev. Lett. 111, 243602 (2013).

[20] See A. J. Leggett, S. Chakravarty, A. T. Dorsey, M. P. A. Fisher, A. Garg, and W. Zwerger, Rev. Mod. Phys. 59, 1 (1987), and references therein.

[21] R. Bulla, N.-H. Tong, and M. Vojta, Phys. Rev. Lett. 91, 170601 (2003); F. B. Anders, R. Bulla, and M. Vojta, ibid. 98, 210402 (2007).

[22] E. Sánchez-Burillo, J. J. García-Ripoll, L. Martín-Moreno, and D. Zueco, Faraday Discuss. 178, 335 (2015).

[23] E. Sanchez-Burillo, D. Zueco, J. J. Garcia-Ripoll, and L. MartinMoreno, Phys. Rev. Lett. 113, 263604 (2014).

[24] I. G. Lang and Y. A. Firsov, Zh. Eksp. Teor. Fiz. 43, 1843 (1962); see also Y. A. Firsov, in Polarons in Advanced Materials, edited by A. S. Alexandrov, Springer Series in Materials Science Vol. 103 (Springer, Dordrecht, 2007).

[25] V. J. Emery and A. Luther, Phys. Rev. B 9, 215 (1974).

[26] W. Zwerger, Z. Phys. B 53, 53 (1983).

[27] R. Silbey and R. A. Harris, J. Chem. Phys. 80, 2615 (1984); R. A. Harris and R. Silbey, ibid. 83, 1069 (1985).

[28] See M. Vojta, Philos. Mag. 86, 1807 (2006), and references therein.

[29] C. Cohen-Tannoudji, J. Dupont-Roc, and G. Grynberg, AtomPhoton Interactions: Basic Process and Applications (WileyVCH, Weinheim, 2004).

[30] V. Weisskopf and E. Wigner, Z. Phys. 63, 54 (1930).

[31] E. Fermi, Rev. Mod. Phys. 4, 87 (1932).

[32] R. H. Dicke, Phys. Rev. 93, 99 (1954).

[33] S. Chakravarty, Phys. Rev. Lett. 49, 681 (1982).

[34] A. J. Bray and M. A. Moore, Phys. Rev. Lett. 49, 1545 (1982).

[35] S. Chakravarty and A. J. Leggett, Phys. Rev. Lett. 52, 5 (1984).

[36] F. Guinea, V. Hakim, and A. Muramatsu, Phys. Rev. B 32, 4410 (1985).
[37] M. Fannes, B. Nachtergaele, and R. F. Werner, Europhys. Lett. 10, 633 (1989).

[38] S. R. White, Phys. Rev. Lett. 69, 2863 (1992).

[39] S. Östlund and S. Rommer, Phys. Rev. Lett. 75, 3537 (1995); J. Dukelsky. M. A. Martín-Delgado, T. Nishino, and G. Sierra, Europhys. Lett. 43, 457 (1998).

[40] I. Affleck, T. Kennedy, E. H. Lieb, and H. Tasaki, Phys. Rev. Lett. 59, 799 (1987).

[41] A. Chin and M. Turlakov, Phys. Rev. B 73, 075311 (2006).

[42] A. W. Chin, J. Prior, S. F. Huelga, and M. B. Plenio, Phys. Rev. Lett. 107, 160601 (2011); S. Bera, A. Nazir, A. W. Chin, H. U. Baranger, and S. Florens, Phys. Rev. B 90, 075110 (2014).

[43] D. P. S. McCutcheon, A. Nazir, S. Bose, and A. J. Fisher, Phys. Rev. B 81, 235321 (2010).

[44] P. Pfeuty, Ann. Phys. (NY) 57, 79 (1970).

[45] A. Kurcz, A. Bermudez, and J. J. García-Ripoll, Phys. Rev. Lett. 112, 180405 (2014); A. Kurcz, J. J. García-Ripoll, and A. Bermudez, Eur. Phys. J. Spec. Top. 224, 483 (2015).

[46] P. Kramer and M. Saraceno, Geometry of the Time-Dependent Variational Principle in Quantum Mechanics (Springer, Berlin, 1981).

[47] N. Wu, L. Duan, X. Li, and Y. Zhao, J. Chem. Phys. 138, 084111 (2013); Y. Yao, L. Duan, Z. Lü, C.-Q. Wu, and Y. Zhao, Phys. Rev. E 88, 023303 (2013).

[48] A. Weichselbaum, F. Verstraete, U. Schollwöck, J. I. Cirac, and J. von Delft, Phys. Rev. B 80, 165117 (2009).

[49] B. Pirvu, V. Murg, J. I. Cirac, and F. Verstraete, New J. Phys. 12, 025012 (2010).

[50] J. J. García-Ripoll, New J. Phys. 8, 305 (2006).

[51] H. Zheng, Eur. Phys. J. B 38, 559 (2004); Q. Wang, A.-Y. Hu, and H. Zheng, Phys. Rev. B 80, 214301 (2009).

[52] K. Bernardet, G. G. Batrouni, J.-L. Meunier, G. Schmid, M. Troyer, and A. Dorneich, Phys. Rev. B 65, 104519 (2002).

[53] C. Aslangul, N. Pottier, and D. Saint-James, J. Phys. (Paris) 47, 1657 (1986).

[54] G. Díaz-Camacho, D. Porras, and J. J. García-Ripoll, Phys. Rev. A 91, 063828 (2015).

[55] L. Van Hove, Physica 21, 901 (1955).

[56] L. Davidovich and H. M. Nussenzveig, in Foundations of Radiation Theory and Quantum Electrodynamics, edited by A. O. Barut (Springer, Berlin, 1980), pp 83-108.

[57] G. M. Palma, K.-A. Suominen, and A. K. Ekert, Proc. R. Soc. London A 452, 567 (1996).

[58] A. González-Tudela, D. Martin-Cano, E. Moreno, L. MartinMoreno, C. Tejedor, and F. J. Garcia-Vidal, Phys. Rev. Lett. 106, 020501 (2011).

[59] A. González-Tudela and D. Porras, Phys. Rev. Lett. 110, 080502 (2013)

[60] P. W. Milonni and P. L. Knight, Phys. Rev. A 10, 1096 (1974).

[61] J. Jünemann, A. Cadarso, D. Pérez-García, A. Bermudez, and J. J. García-Ripoll, Phys. Rev. Lett. 111, 230404 (2013). 